\title{
Regularity Results for a Nonlinear Elliptic-Parabolic System with Oscillating Coefficients
}

\author{
Xiangsheng $\mathrm{Xu}^{*}$ \\ Department of Mathematics $\mathcal{E}$ Statistics, Mississippi State University, Mississippi \\ State, MS 39762, USA
}

Received 9 May 2020; Accepted (in revised version) 4 January 2021

\begin{abstract}
In this paper we study the initial boundary value problem for the system $\operatorname{div}(\sigma(u) \nabla \varphi)=0, u_{t}-\Delta u=\sigma(u)|\nabla \varphi|^{2}$. This problem is known as the thermistor problem which models the electrical heating of conductors. Our assumptions on $\sigma(u)$ leave open the possibility that $\liminf _{u \rightarrow \infty} \sigma(u)=0$, while $\limsup _{u \rightarrow \infty} \sigma(u)$ is large. This means that $\sigma(u)$ can oscillate wildly between 0 and a large positive number as $u \rightarrow \infty$. Thus our degeneracy is fundamentally different from the one that is present in porous medium type of equations. We obtain a weak solution $(u, \varphi)$ with $|\nabla \varphi|,|\nabla u| \in$ $L^{\infty}$ by first establishing a uniform upper bound for $e^{\varepsilon u}$ for some small $\varepsilon$. This leads to an inequality in $\nabla \varphi$, from which the regularity result follows. This approach enables us to avoid first proving the Hölder continuity of $\varphi$ in the space variables, which would have required that the elliptic coefficient $\sigma(u)$ be an $A_{2}$ weight. As it is known, the latter implies that $\ln \sigma(u)$ is "nearly bounded".
\end{abstract}

Key Words: Oscillating coefficients, the thermistor problem, quadratic nonlinearity.

AMS Subject Classifications: 35B45, 35B65, 35M33, 35Q92

\section{Introduction}

Let $\Omega$ be a bounded domain in $\mathbb{R}^{N}$ with sufficiently smooth boundary $\partial \Omega$ and $T$ any positive number. We consider the initial boundary value problem

$$
\begin{array}{ll}
u_{t}-\Delta u=\sigma(u)|\nabla \varphi|^{2} & \text { in } \Omega_{T}, \\
\operatorname{div}(\sigma(u) \nabla \varphi)=0 & \text { in } \Omega_{T}, \\
u=u_{0} & \text { on } \partial_{p} \Omega_{T}, \\
\varphi=\varphi_{0} & \text { on } \Sigma_{T},
\end{array}
$$

${ }^{*}$ Corresponding author. Email addresses: xxu@math.msstate. edu (X. Xu) 
where

$$
\begin{aligned}
& \Omega_{T}=\Omega \times(0, T), \\
& \Sigma_{T}=\partial \Omega \times(0, T), \quad \text { the lateral boundary of } \Omega_{T}, \\
& \partial_{p} \Omega_{T}=\Sigma_{T} \cup \Omega \times\{0\}, \quad \text { the parabolic boundary of } \Omega_{T} .
\end{aligned}
$$

We are interested in the regularity properties of weak solutions when the elliptic coefficient $\sigma(u)$ in the second equation may become oscillatory as $u \rightarrow \infty$. To be precise, we establish the following

Theorem 1.1 (Main Theorem). Assume:

(H1) the function $\sigma$ is continuously differentiable on the interval $[0, \infty)$ with

$$
\begin{array}{ll}
c_{0} e^{-\beta s} \leq \sigma(s) \leq c_{1} & \text { on }[0, \infty) \text { for some } c_{0}, c_{1}, \beta \in(0, \infty), \\
\left|\sigma^{\prime}(s)\right| \leq c_{2} e^{\gamma s} & \text { on }[0, \infty) \text { for some } c_{2}, \gamma \in(0, \infty),
\end{array}
$$

(H2) $u_{0}, \varphi_{0} \in C\left([0, T] ; C^{1}(\bar{\Omega})\right)$ with $\left.u_{0}\right|_{\partial_{p} \Omega_{T}} \geq 0$ and $\partial_{t} u_{0}-\Delta u_{0} \in L^{s}\left(\Omega_{T}\right), \Delta \varphi_{0} \in$ $L^{\infty}\left(0, T ; L^{s}(\Omega)\right)$ for each $s>1$,

(H3) $\partial \Omega$ is $C^{1,1}$.

Then there is a unique weak solution $(u, \varphi)$ to (1.1a)-(1.1d) with $u \geq 0$ and

$$
\nabla u, \nabla \varphi \in L^{\infty}\left(\Omega_{T}\right)
$$

The notion of a weak solution is defined as follows:

Definition 1.1. We say that $(u, \varphi)$ is a weak solution to (1.1a)-(1.1d) if

(D1) $u, \varphi \in L^{2}\left(0, T ; W^{1,2}(\Omega)\right)$,

(D2) $u=u_{0}, \varphi=\varphi_{0}$ on $\Sigma_{T}$ in the sense of the trace theorem and

$$
\begin{aligned}
& -\int_{\Omega_{T}} u \xi_{t} d x d t+\int_{\Omega_{T}} \nabla u \nabla \xi d x d t \\
& \quad=\int_{\Omega_{T}} \sigma(u)|\nabla \varphi|^{2} d x d t+\int_{\Omega} u_{0}(x, 0) \xi(x, 0) d x, \\
& \int_{\Omega_{T}} \sigma(u) \nabla \varphi \nabla \eta d x d t=0,
\end{aligned}
$$

for each pair of smooth functions $\xi, \eta$ with $\xi=\eta=0$ on $\Sigma_{T}$ and $\xi(x, T)=\eta(x, T)=0$. 
We quickly offer another perspective on the initial condition for $u$ in the weak solution. The weak maximum principle asserts that

$$
\|\varphi\|_{\infty, \Omega_{T}} \leq\left\|\varphi_{0}\right\|_{\infty, \Omega_{T}}
$$

We can easily derive from $(1.1 b)$ that

$$
\int_{\Omega_{T}} \sigma(u)|\nabla \varphi|^{2} \xi d x d t=-\int_{\Omega_{T}} \sigma(u) \varphi \nabla \varphi \nabla \xi d x d t \quad \text { for each } \xi \text { with }\left.\xi\right|_{\Sigma_{T}}=0 .
$$

This together with (1.1a) implies that $u_{t} \in L^{2}\left(0, T ; W^{-1,2}(\Omega)\right)$. Thus we can conclude that $u \in C\left([0, T], L^{2}(\Omega)\right)$. The initial condition $u(x, 0)=u_{0}(x, 0)$ can also be understood to hold in this space. Of course, under (1.4), we have $u_{t} \in L^{s}\left(\Omega_{T}\right)$ for each $s>1$. Also, the proof of the uniqueness of a weak solution under (1.4) is rather straightforward. We shall omit it here.

Physically, problem (1.1a)-(1.1d) may be proposed as a model for the electrical heating of a conductor, the so-called thermistor problem. In this case $u$ is the temperature and $\varphi$ the electrical potential of the conductor. The heat source is the Joule heating $\sigma(u) \nabla \varphi \cdot \nabla \varphi$, where $\sigma(u)$ is the temperature-dependent electrical conductivity. We have taken the thermal conductivity to be 1 . There is a large body of literature devoted to the study of (1.1a)(1.1d) under various assumptions on $\sigma(s)$ and the boundary conditions, and also various generalizations of the problem. For the mathematical analysis of the associated stationary problem, we would like to mention $[6,7,11]$. Modeling and numerical simulations were investigated in $[2,13,19]$. For optimal control issues, we refer the reader to [12] and the references therein. Also see [1] and its references for obstacle thermistor problems. Of course, there are many more papers that we have failed to mention, and it is simply beyond the scope of this paper to give a comprehensive review of the current research in this area.

A very important issue about the time-dependent problem is: How does one prevent the thermal run-away from occurring? The blow-up of solutions was studied in [3]. In applications, blow-up of solutions are not welcome in general. Thus we will focus our attention on the boundedness of $u$. If $\sigma$ is also bounded away from 0 below, then (1.1b) becomes uniformly elliptic and one has

$$
\varphi \in L^{\infty}\left(0, T ; C^{\alpha}(\bar{\Omega})\right) \text { for some } \alpha \in(0,1) .
$$

This combined with a result in [30] asserts that $u$ is Hölder continuous in $\overline{\Omega_{T}}$. With the aid of this, we can conclude from [16, p. 82] that for each $p>1$ there is a positive number $c$ depending on the continuity of $\sigma(u)$ and $C^{1,1}$ boundary such that

$$
\|\nabla \varphi\|_{p, \Omega_{T}} \leq c\left\|\nabla \varphi_{0}\right\|_{p, \Omega_{T}} .
$$

By the proof of Lemma 3.3 below, (1.9) implies $|\nabla u| \in L^{\infty}\left(\Omega_{T}\right)$. Now write (1.1b) in the form

$$
\Delta \varphi=-\frac{\sigma^{\prime}(u)}{\sigma(u)} \nabla u \cdot \nabla \varphi .
$$


This puts us in a position to apply the classical Calderón-Zygmund estimate [5]. Upon doing so, we establish (1.4).

Under (H1), the problem immediately becomes very delicate because we have to leave open the possibility that $u$ is not bounded above. The reason is simple: The term on the right-hand of (1.1a) is only an $L^{1}$ function from the usual energy estimates. Consequently, (1.1b) could become degenerate and a priori estimates are difficult to obtain. In fact, even an $L^{p}, p \geq 1$, estimate for $\nabla \varphi$ is unlikely unless additional assumptions on $\sigma$ are made $[20,21]$. Furthermore, assumption (H1) allows the possibility that

$$
\limsup _{s \rightarrow \infty} \sigma(s)>0 \text { and } \quad \liminf _{s \rightarrow \infty} \sigma(s)=0
$$

hold simultaneously. This means that the function $\sigma(s)$ can oscillate wildly between 0 and a positive number as $s \rightarrow \infty$. We can easily come up with an example of such functions. Say,

$$
\sigma(s)=c_{3}\left(1+\sin e^{\gamma s}\right)+c_{0} e^{-\beta s}, \quad c_{3}>0 .
$$

By virtue of the classical regularity theory [10] for degenerate and/or singular elliptic equations of the type (1.1b), $\sigma(u)$ must be an $A_{2}$-weight for (1.8) to hold. We say that $\sigma(u)$ is an $A_{2}$-weight if there is a positive number $c$ such that

$$
\underset{B_{r}(y)}{f} \sigma(u) d x \underset{B_{r}(y)}{f} \frac{1}{\sigma(u)} d x \leq c \quad \text { for all } y \in \Omega, r>0 \text { with } B_{r}(y) \subset \Omega,
$$

where $B_{r}(y)$ denotes the open ball centered at $y$ with radius $r$. A theorem in [18, p. 141] asserts that a function $f$ is an $A_{2}$ weight if and only if $\ln f$ belongs to BMO. The latter implies that over any ball, the average oscillation of $\ln f$ must be bounded. In the situation considered here, to obtain (1.12) we have to assume $\sigma(u)=e^{-c u}$ for some $c>0$ according to a result in [23]. In general, our main theorem seems to lie outside the scope of [10]. This is the main motivation for our study. In a series of three papers [20-22], the author obtained the boundedness of $u$ under the assumptions that the given function $\sigma(s)$ has the properties:

(C1) $\sigma(s)$ is continuous, positive, and bounded above;

(C2) $\lim _{s \rightarrow \infty} \sigma(s)=0$; and

(C3) $\lim _{\tau \rightarrow 0^{+}} \frac{\sigma(s+\tau)}{\sigma(s)}=1$ uniformly on $[0, \infty)$.

In particular, condition (C2) is essential to the argument there. We have managed to remove this condition here, thereby allowing oscillation in $\sigma$. A result in [22] asserts that (C3) implies that $\sigma(u)$ is bounded below by an exponential function. Thus we have also weaken (C3) substantially. The trade-off for us is that we have to assume that $\sigma$ is continuously differentiable. 
Recall that solutions to the initial boundary value problem for the equation $u_{t}-\Delta u=$ $\sigma(u)$ can blow up in finite time when $\sigma(u)$ is superlinear, i.e.,

$$
\lim _{u \rightarrow \infty} \frac{\sigma(u)}{u}=\infty, \quad \int^{\infty} \frac{1}{\sigma(u)} d u<\infty
$$

See, for example, [4]. It would be interesting to know if we can allow $\sigma(u)$ to be bounded above by a linear function.

The difficult features in our problem are the possible oscillation of $\sigma(u)$ and the exponential growth conditions we impose on $\frac{1}{\sigma}, \sigma^{\prime}$. They prevent us from employing the traditional approach of going from lower regularity to higher one. Instead, we will prove (1.4) directly. This is done by obtaining a uniform upper bound for $e^{\varepsilon u}$ for $\varepsilon$ sufficiently small. The idea is motivated by a recent paper of the author [24]. Then we bound $\nabla u$ by $\nabla \varphi$, and vice versa, thereby establishing an inequality in $\|\nabla \varphi\|_{\infty, \Omega_{T}}$. This enables us to prove existence for $T$ suitably small. Then we further show that we can extend our solution in the time direction as far away as we want.

This work is organized as follows. Section 2 is largely preparatory. We collect some relevant known results. The proof of the main theorem is contained in Section 3.

We follow the well-established notation convention whenever possible. Therefore, throughout this paper, the letter $c$ will be used to denote a positive number that depends only on the given data unless stated otherwise. The dot product of two column vectors F, G is denoted by F . G. When we apply the Sobolev embedding theorem, we only deal with the case $N>2$. The case $N=2$ can be handled similarly.

\section{Preliminaries}

In this section we collect some known results for later use. We begin with Grönwall's inequality.

Lemma 2.1. Suppose that a differentiable function $h(t)$ satisfies the inequality

$$
h^{\prime}(t) \leq \operatorname{ch}(t)+g(t) \text { on }[0, \infty),
$$

where $c$ is a constant and $g(t)$ a locally integrable function. Then

$$
h(t) \leq h(0) e^{c t}+\int_{0}^{t} g(\tau) e^{c(t-\tau)} d \tau .
$$

We also need the interpolation inequality

$$
\|u\|_{q} \leq \varepsilon\|u\|_{r}+\varepsilon^{-\mu}\|u\|_{\ell}
$$

where $1 \leq \ell \leq q \leq r$ with $\mu=\left(\frac{1}{\ell}-\frac{1}{q}\right) /\left(\frac{1}{q}-\frac{1}{r}\right)$.

The next two lemmas deals with sequences of nonnegative numbers which satisfy certain recursive inequalities. 
Lemma 2.2. Let $\left\{y_{n}\right\}, n=0,1, \cdots$, be a sequence of positive numbers satisfying the recursive inequalities

$$
y_{n+1} \leq c b^{n} y_{n}^{1+\alpha} \text { for some } b>1, c, \alpha \in(0, \infty) .
$$

If

$$
y_{0} \leq c^{-\frac{1}{\alpha}} b^{-\frac{1}{\alpha^{2}}}
$$

then $\lim _{n \rightarrow \infty} y_{n}=0$.

This lemma can be found in ( $[8, \mathrm{p} .12])$

Lemma 2.3. Let $h(\tau)$ be a continuous non-negative function defined on $\left[0, T_{0}\right]$ for some $T_{0}>0$. Suppose that there exist three positive numbers $\varepsilon, \delta, b$ such that

$$
h(\tau) \leq \varepsilon h^{1+\delta}(\tau)+b \quad \text { for each } \tau \in\left[0, T_{0}\right] .
$$

Then $h(\tau) \leq \frac{1}{[\varepsilon(1+\delta)]^{\frac{1}{\delta}}}$ for each $\tau \in\left[0, T_{0}\right]$, provided that

$$
\varepsilon \leq \frac{\delta^{\delta}}{(b+\delta)^{\delta}(1+\delta)^{1+\delta}} \quad \text { and } \quad h(0) \leq \frac{1}{[\varepsilon(1+\delta)]^{\frac{1}{\delta}}} \equiv s_{0} .
$$

This lemma has played a key role in the proof of our main theorem. It can be viewed as a continuous version of Lemma 3.1 in $[15,17]$. Its proof is elementary and can be found in [29].

\section{Proof of the main result}

The proof of the main theorem is divided into several lemmas. We assume that (1.1a)(1.1d) has a weak solution $(u, \varphi)$ with $u \in L^{\infty}\left(\Omega_{T}\right)$. By our discussion in the introduction, this actually implies (1.4) and more. We will indicate how we obtain such an (approximate) solution via the Leray-Schauder fixed point theorem near the end of the section. We shall begin with the exponential integrability of $u$ [28].

Lemma 3.1. For each $m \in\left(0, \frac{1}{c_{1}\left\|\varphi_{0}\right\|_{\infty, \Omega}}\right)$ there is a positive number $c$ such that

$$
\sup _{0 \leq t \leq T} \int_{\Omega} e^{m u} d x+\int_{\Omega_{T}}\left(e^{m u}|\nabla u|^{2}+\sigma(u) e^{m u}|\nabla \varphi|^{2}\right) d x d t \leq c .
$$

Proof. The weak maximum principle asserts that

$$
\|\varphi\|_{\infty, \Omega} \leq\left\|\varphi_{0}\right\|_{\infty, \Omega}
$$

We use $\varphi-\varphi_{0}$ as a test function in (1.1b) to obtain

$$
\int_{\Omega} \sigma(u)|\nabla \varphi|^{2} d x \leq \int_{\Omega} \sigma(u)\left|\nabla \varphi_{0}\right|^{2} d x \leq c .
$$


On the other hand, use $u-u_{0}$ as a test function in (1.1a) to derive

$$
\begin{aligned}
& \frac{1}{2} \frac{d}{d t} \int_{\Omega}\left(u-u_{0}\right)^{2} d x+\int_{\Omega}\left|\nabla\left(u-u_{0}\right)\right|^{2} d x \\
= & \int_{\Omega} \sigma(u)|\nabla \varphi|^{2} d x+\int_{\Omega}\left(-\partial_{t} u_{0}+\Delta u_{0}\right)\left(u-u_{0}\right) d x \\
= & -\int_{\Omega} \sigma(u) \varphi \nabla \varphi \nabla\left(u-u_{0}\right) d x-\int_{\Omega} \nabla u_{0} \nabla\left(u-u_{0}\right) d x-\int_{\Omega} \partial_{t} u_{0}\left(u-u_{0}\right) d x \\
\leq & \frac{1}{2} \int_{\Omega}\left|\nabla\left(u-u_{0}\right)\right|^{2} d x+\frac{1}{2} \int_{\Omega}\left(u-u_{0}\right)^{2} d x+c+c \int_{\Omega}\left(\left(\partial_{t} u_{0}\right)^{2}+\left|\nabla u_{0}\right|^{2}\right) d x .
\end{aligned}
$$

Use Grönwall's inequality to yield

$$
\sup _{0 \leq t \leq T} \int_{\Omega} u^{2} d x+\int_{\Omega_{T}}|\nabla u|^{2} d x d \tau \leq c e^{T}+c T+c .
$$

Fix

$$
K \geq\left\|u_{0}\right\|_{\infty, \Omega_{T}}
$$

For any $C^{1}$ function $f$ on $\mathbb{R}$ with

$$
f>0 \text { and } f^{\prime}>0,
$$

we use $(f(u)-f(K))^{+}$as a test function in (1.1a) to obtain

$$
\frac{d}{d t} \int_{\Omega} \int_{0}^{u}(f(s)-f(K))^{+} d s d x+\int_{\{u \geq K\}}\left(f^{\prime}(u)|\nabla u|^{2}+\sigma(u) \varphi \nabla \varphi f^{\prime}(u) \nabla u\right) d x=0 .
$$

On the other hand, use $(f(u)-f(K))^{+} \varphi$ as a test function in (1.1a) to yield

$$
\begin{aligned}
& \int_{\{u \geq K\}}\left(f(u) \sigma(u)|\nabla \varphi|^{2}+\sigma(u) \varphi \nabla \varphi f^{\prime}(u) \nabla u\right) d x \\
= & f(K) \int_{\{u \geq K\}} \sigma(u)|\nabla \varphi|^{2} d x \leq c f(K) .
\end{aligned}
$$

Combing the preceding two equations, we arrive at

$$
\begin{aligned}
& \quad \frac{d}{d t} \int_{\Omega} \int_{0}^{u}(f(s)-f(K))^{+} d s d x+\varepsilon \int_{\{u \geq K\}}\left(f^{\prime}(u)|\nabla u|^{2}+f(u) \sigma(u)|\nabla \varphi|^{2}\right) d x \\
& \quad+\int_{\{u \geq K\}}\left((1-\varepsilon) f^{\prime}(u)|\nabla u|^{2}+2 \sigma(u) \varphi \nabla \varphi f^{\prime}(u) \nabla u+(1-\varepsilon) f(u) \sigma(u)|\nabla \varphi|^{2}\right) d x \\
& \leq c f(K),
\end{aligned}
$$

where $\varepsilon \in(0,1)$. The last integrand in the above inequality is non-negative if $f$ is so chosen that

$$
\frac{f^{\prime}(u)}{f(u)} \leq \frac{(1-\varepsilon)^{2}}{c_{1}\left\|\varphi_{0}\right\|_{\infty, \Omega}^{2}} \leq \frac{(1-\varepsilon)^{2}}{\sigma(u) \varphi^{2}} .
$$


We take

$$
f(s)=e^{m u} .
$$

For (3.6)to hold for $\varepsilon$ sufficiently small, it is enough to take

$$
m<\frac{1}{c_{1}\left\|\varphi_{0}\right\|_{\infty, \Omega}^{2}} .
$$

Use this in (3.5), integrate, and keep in mind (3.4) to derive the desired result. The proof is completed.

We would like to remark that if

$$
T \leq 1,
$$

then the constant $c$ in (3.1) can be made independent of $T$. This can be easily seen from (3.4). For this purpose only, we will assume (3.7) from here on.

Now let

$$
w=e^{\varepsilon u}, \quad \varepsilon \in(0,1) .
$$

Then $w$ satisfies the problem

$$
\begin{array}{ll}
w_{t}-\Delta w=\varepsilon \sigma(u)|\nabla \varphi|^{2} w-\varepsilon^{2} e^{\varepsilon u}|\nabla u|^{2} \leq \varepsilon \sigma(u)|\nabla \varphi|^{2} w & \text { in } \Omega_{T}, \\
w=e^{\varepsilon u_{0}} & \text { on } \partial_{p} \Omega_{T} .
\end{array}
$$

Lemma 3.2. Let $w$ be given as above. For each $\frac{N+2}{N}>\ell>1$ and $0<\varepsilon<\min \left\{1, \frac{1}{2 c_{1} \ell\left\|\varphi_{0}\right\|_{\infty, \Omega}^{2}}\right\}$, there is a positive number $c$ such that

$$
\|w\|_{\infty, \Omega_{T}}=\left\|e^{\varepsilon u}\right\|_{\infty, \Omega_{T}} \leq c T^{\frac{1}{2 \ell}}\|\nabla \varphi\|_{\frac{2 \ell}{\ell-1}, \Omega_{T}}^{\frac{N+2}{N+2 \ell}}+c .
$$

Proof. Let

$$
\frac{k}{2} \geq \max \left\{1,\left\|e^{u_{0}}\right\|_{\infty, \Omega_{T}}\right\}
$$

be selected as below. Set

$$
k_{n}=k-\frac{k}{2^{n+1}}, \quad n=0,1, \cdots
$$

Then we have

$$
\left.\left(w-k_{n}\right)^{+}\right|_{\partial_{p} \Omega_{T}}=0 .
$$

Use $\left(w-k_{n+1}\right)^{+}$as a test function in (3.8a) to obtain

$$
\begin{aligned}
& \frac{1}{2} \frac{d}{d t} \int_{\Omega}\left[\left(w-k_{n+1}\right)^{+}\right]^{2} d x+\int_{\Omega}\left|\nabla\left(w-k_{n+1}\right)^{+}\right|^{2} d x \\
= & \int_{\Omega} \varepsilon \sigma(u)|\nabla \varphi|^{2} w\left(w-k_{n+1}\right)^{+} d x \leq\left. c \int_{\Omega} \nabla \varphi\right|^{2} w\left(w-k_{n+1}\right)^{+} d x .
\end{aligned}
$$


Integrate to get

$$
\begin{aligned}
& \max _{0 \leq t \leq T} \int_{\Omega}\left[\left(w-k_{n+1}\right)^{+}\right]^{2} d x+\int_{\Omega_{T}}\left|\nabla\left(w-k_{n+1}\right)^{+}\right|^{2} d x d t \\
& \leq c\|\nabla \varphi\|_{\frac{2 \ell}{\ell-1}, \Omega_{T}}^{2}\left(\int_{\Omega_{T}}\left(w\left(w-k_{n+1}\right)^{+}\right)^{\ell} d x d t\right)^{\frac{1}{\ell}},
\end{aligned}
$$

where

$$
1<\ell<\frac{2}{N}+1
$$

Let

$$
y_{n}=\left(\int_{\Omega_{T}}\left[\left(w-k_{n}\right)^{+}\right]^{2 \ell} d x d t\right)^{\frac{1}{\ell}} .
$$

Assume $N>2$. We estimate from the Sobolev embedding theorem that

$$
\begin{aligned}
& \int_{0}^{T} \int_{\Omega}\left[\left(w-k_{n+1}\right)^{+}\right]^{\frac{4}{N}+2} d x d t \\
\leq & \int_{0}^{T}\left(\int_{\Omega}\left[\left(w-k_{n+1}\right)^{+}\right]^{2} d x\right)^{\frac{2}{N}}\left(\int_{\Omega}\left[\left(w-k_{n+1}\right)^{+}\right]^{\frac{2 N}{N-2}} d x\right)^{\frac{N-2}{N}} d t \\
\leq & c\left(\max _{0 \leq t \leq T} \int_{\Omega}\left[\left(w-k_{n+1}\right)^{+}\right]^{2} d x\right)^{\frac{2}{N}} \int_{0}^{T} \int_{\Omega}\left[\nabla\left(w-k_{n+1}\right)^{+}\right]^{2} d x d t \\
\leq & c\left\|\sigma(u)|\nabla \varphi|^{2}\right\|_{\frac{\ell}{\ell-1}, \Omega_{T}}^{1+\frac{2}{N}}\left(\int_{\Omega_{T}}\left(w\left(w-k_{n+1}\right)^{+}\right)^{\ell} d x d t\right)^{\frac{N+2}{N \ell}} .
\end{aligned}
$$

Set

$$
A_{n+1}=\left\{w \geq k_{n+1}\right\} .
$$

This combined with (3.12) gives

$$
\begin{aligned}
y_{n+1} & =\left(\int_{\Omega_{T}}\left[\left(w-k_{n+1}\right)^{+}\right]^{2 \ell} d x d t\right)^{\frac{1}{\ell}} \\
& \leq\left(\int_{\Omega_{T}}\left[\left(w-k_{n+1}\right)^{+}\right]^{2 \frac{N+2}{N}} d x d t\right)^{\frac{N}{N+2}}\left|A_{n+1}\right|^{\frac{1}{\ell}-\frac{N}{N+2}} \\
& \leq c\left\|\sigma(u)|\nabla \varphi|^{2}\right\|_{\frac{\ell}{\ell-1}, \Omega_{T}}\left(\int_{\Omega_{T}}\left(w\left(w-k_{n+1}\right)^{+}\right)^{\ell} d x d t\right)^{\frac{1}{\ell}}\left|A_{n+1}\right|^{\frac{1}{\ell}-\frac{N}{N+2}} .
\end{aligned}
$$


On the other hand, we have

$$
\begin{aligned}
y_{n} & \geq\left(\int_{A_{n+1}}\left[\left(w-k_{n}\right)^{+}\right]^{2 \ell} d x d t\right)^{\frac{1}{\ell}} \\
& =\left(\int_{A_{n+1}} w^{\ell}\left[\left(w-k_{n}\right)^{+}\right]^{\ell}\left(1-\frac{k_{n}}{w}\right)^{\ell} d x d t\right)^{\frac{1}{\ell}} \\
& \geq\left(\int_{A_{n+1}} w^{\ell}\left[\left(w-k_{n}\right)^{+}\right]^{\ell}\left(1-\frac{k_{n}}{k_{n+1}}\right)^{\ell} d x d t\right)^{\frac{1}{\ell}} \\
& \geq \frac{1}{2^{(n+2)}}\left(\int_{A_{n+1}} w^{\ell}\left[\left(w-k_{n}\right)^{+}\right]^{\ell} d x d t\right)^{\frac{1}{\ell}} .
\end{aligned}
$$

Furthermore,

$$
y_{n} \geq\left(k_{n+1}-k_{n}\right)^{2}\left|A_{n+1}\right|^{\frac{1}{\ell}}=\frac{k^{2}}{2^{2(n+2)}}\left|A_{n+1}\right|^{\frac{1}{\ell}} .
$$

Finally, we arrive at

$$
\begin{aligned}
y_{n+1} & \leq c 2^{(n+2)}\left\|\sigma(u)|\nabla \varphi|^{2}\right\|_{\frac{\ell}{\ell-1}, \Omega_{T}} y_{n}\left|A_{n+1}\right|^{\frac{1}{\ell}-\frac{N}{N+2}} \\
& \leq\left.\frac{c b^{n}}{k^{2 \frac{N+2-N \ell}{N+2}}}|\sigma(u)| \nabla \varphi\right|^{2} \|_{\frac{\ell}{\ell-1}, \Omega_{T}} y_{n}^{1+\frac{N+2-N \ell}{N+2}}
\end{aligned}
$$

where $b>1$. Thus by Lemma 2.2 , if we take $k$ so that

$$
y_{0} \leq c\left(\frac{k^{2 \frac{N+2-N \ell}{N+2}}}{\left\|\sigma(u)|\nabla \varphi|^{2}\right\|_{\frac{\ell}{\ell-1}, \Omega_{T}}}\right)^{\frac{N+2}{N+2-N \ell}},
$$

then

$$
w \leq k
$$

Taking into account of (3.10), it is enough for us to take

$$
k=c y_{0}^{\frac{1}{2}}\left\|\sigma(u)|\nabla \varphi|^{2}\right\|_{\frac{\ell}{\ell-1}, \Omega_{T}}^{\frac{N+2-N \ell)}{2(+2)}}+2 e^{\left\|u_{0}\right\|_{\infty, \Omega_{T}} .}
$$

Choose $\varepsilon$ suitably small so that

$$
2 \ell \varepsilon<\frac{1}{c_{1}\left\|\varphi_{0}\right\|_{\infty, \Omega}^{2}}
$$

By Lemma 3.1, we have

$$
y_{0} \leq\left(\int_{\Omega_{T}} e^{2 \ell \varepsilon u} d x d t\right)^{\frac{1}{\ell}} \leq c T^{\frac{1}{\ell}} .
$$

Plug this into (3.18) to get the desired result. 
Lemma 3.3. For each $r \in(N, N+1)$ there is a positive number $c$ such that

$$
\|\nabla u\|_{\infty, \Omega_{T}} \leq c T^{\frac{r}{2}-\frac{N}{2}}\|\nabla \varphi\|_{\infty, \Omega_{T}}^{2}+c .
$$

Proof. Consider the function

$$
G=\frac{1}{(4 \pi)^{\frac{N}{2}}} \int_{0}^{t} \frac{1}{(t-\tau)^{\frac{N}{2}}} \int_{\mathbb{R}^{N}} \exp \left(-\frac{|x-y|^{2}}{4(t-\tau)}\right) \sigma(u)|\nabla \varphi|^{2} \chi_{\Omega} d y d \tau .
$$

We see from ( [14], Chapter IV) that G satisfies

$$
\begin{array}{ll}
G_{t}-\Delta G=\sigma(u)|\nabla \varphi|^{2} \chi_{\Omega} & \text { in } \mathbb{R}^{N} \times(0, T), \\
G(x, 0)=0 & \text { on } \mathbb{R}^{N} .
\end{array}
$$

Furthermore, for each $p>1$ there is a positive number $c$ such that

$$
\left\|G_{t}\right\|_{p, \Omega_{T}}+\|G\|_{L^{p}\left(0, T ; W^{2, p}(\Omega)\right)} \leq c\left\|\sigma(u)|\nabla \varphi|^{2}\right\|_{p, \Omega_{T}} .
$$

Set

$$
s=\frac{|x-y|}{2 \sqrt{t-\tau}} .
$$

Let $r$ be given as in the lemma. Then we have

$$
s^{r} \exp \left(-s^{2}\right) \leq c(r) \text { on }[0, \infty) .
$$

With this in mind, we estimate

$$
\begin{aligned}
|\nabla G| & =\left.\left|\frac{1}{2(4 \pi)^{\frac{N}{2}}} \int_{0}^{t} \frac{1}{(t-\tau)^{\frac{N}{2}}} \int_{\mathbb{R}^{N}}(x-y) \exp \left(-s^{2}\right) \sigma(u)\right| \nabla \varphi\right|^{2} \chi_{\Omega} d y d \tau \mid \\
& \leq\left. c \int_{0}^{t} \frac{1}{(t-\tau)^{1+\frac{N}{2}}} \int_{\mathbb{R}^{N}} \frac{(2 \sqrt{t-\tau})^{r}}{|x-y|^{r-1}} s^{r} \exp \left(-s^{2}\right)|\sigma(u)| \nabla \varphi\right|^{2} \chi_{\Omega} d y d \tau \\
& \leq c\|\nabla \varphi\|_{\infty, \Omega_{T}}^{2} \int_{0}^{t} \frac{1}{(t-\tau)^{1+\frac{N}{2}-\frac{r}{2}}} \int_{\mathbb{R}^{N}} \frac{\chi_{\Omega}}{|x-y|^{r-1}} d y d \tau \\
& \leq c t^{\frac{r}{2}-\frac{N}{2}}\|\nabla \varphi\|_{\infty, \Omega_{T}}^{2} .
\end{aligned}
$$

Obviously, $F \equiv u-G$ satisfies the problem

$$
\begin{array}{ll}
F_{t}-\Delta F=0 & \text { in } \Omega_{T}, \\
F=u_{0}-G & \text { on } \partial_{p} \Omega_{T} .
\end{array}
$$

We can easily conclude from (3.19) and the classical regularity theory for the heat equation [14, Chapter IV], that for $p$ large enough there holds

$$
\begin{aligned}
\|\nabla F\|_{\infty, \Omega_{T}} & \leq c\left\|\nabla u_{0}-\nabla G\right\|_{\infty, \Omega_{T}}+c\left\|\partial_{t} u_{0}-\Delta u_{0}-\partial_{t} G+G \mid\right\|_{p, \Omega_{T}} \\
& \leq c T^{\frac{r}{2}-\frac{N}{2}}\|\nabla \varphi\|_{\infty, \Omega_{T}}^{2}+c .
\end{aligned}
$$


Here we have used (H2). It easily follows that

$$
\|\nabla u\|_{\infty, \Omega_{T}} \leq c T^{\frac{r}{2}-\frac{N}{2}}\|\nabla \varphi\|_{\infty, \Omega_{T}}^{2}+c .
$$

Thus, we complete the proof.

By (H1), we have

$$
\left|\frac{\sigma^{\prime}(u)}{\sigma(u)}\right| \leq c e^{(\beta+\gamma) u} .
$$

Use $\varphi-\varphi_{0}$ as a test function in (1.10) to derive

$$
\int_{\Omega}|\nabla \varphi|^{2} d x \leq \int_{\Omega}\left(e^{(\beta+\gamma) u}|\nabla u|\right)^{2} d x+c .
$$

On account of the classical Calderón-Zygmund estimate, for each $p>0$ there is a positive number $c$ such that

$$
\|\varphi\|_{W^{2, p}(\Omega)} \leq c\left\|e^{(\beta+\gamma) u} \nabla u \cdot \nabla \varphi\right\|_{p, \Omega}+c\left\|\varphi_{0}\right\|_{W^{2, p}(\Omega)} .
$$

Take $p>N$. Then we derive from the Sobolev embedding theorem and (2.1) that

$$
\begin{aligned}
\|\nabla \varphi\|_{\infty, \Omega} & \leq c\|\varphi\|_{W^{2, p}(\Omega)} \\
& \leq c\left\|e^{(\beta+\gamma) u} \nabla u\right\|_{\infty, \Omega}\|\nabla \varphi\|_{p, \Omega}+c \\
& \leq c\left\|e^{(\beta+\gamma) u} \nabla u\right\|_{\infty, \Omega}\left(\varepsilon\|\nabla \varphi\|_{\infty, \Omega}+\frac{1}{\varepsilon^{\frac{p-2}{2}}}\|\nabla \varphi\|_{2, \Omega}\right)+c \\
& =\frac{1}{2}\|\nabla \varphi\|_{\infty, \Omega}+c\left\|e^{(\beta+\gamma) u} \nabla u\right\|_{\infty, \Omega}^{\frac{p-2}{2}}\left(\left\|e^{(\beta+\gamma) u} \nabla u\right\|_{2, \Omega}+c\right)+c .
\end{aligned}
$$

Consequently,

$$
\|\nabla \varphi\|_{\infty, \Omega} \leq c\left\|e^{(\beta+\gamma) u} \nabla u\right\|_{\infty, \Omega}^{\frac{p}{2}}+c .
$$

By Lemmas 3.2 and 3.3,

$$
\begin{aligned}
& \|\nabla \varphi\|_{\infty, \Omega_{T}} \leq c\left\|e^{(\beta+\gamma) u} \nabla u\right\|_{\infty, \Omega_{T}}^{\frac{p}{2}}+c
\end{aligned}
$$

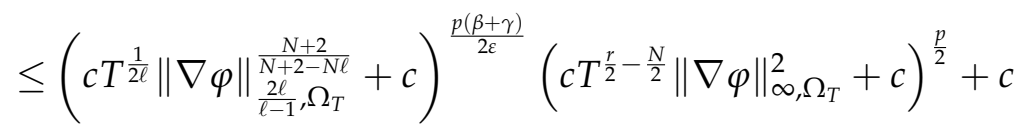

$$
\begin{aligned}
& \leq c T^{a}\|\nabla \varphi\|_{\infty, \Omega_{T}}^{b}+c,
\end{aligned}
$$

where $a, b$ are two positive numbers. Obviously, we can take

$$
b=\frac{(N+2)(\beta+\gamma) p}{2 \varepsilon(N+2-N \ell)}+p>1
$$


In view of (3.7), $a$ is the smallest power of $T$ that appear in the product in (3.28). We will first show $\varphi \in L^{\infty}\left(\Omega_{T}\right)$ for $T$ suitably small [26]. This is achieved via Lemma 2.3. Then extend the solution in the time direction. To this end, remember that $c$ in (3.28) is independent of $T$. Set

$$
\varepsilon=c T^{a} \text {. }
$$

Consider the function $g(\tau)=\varepsilon \tau^{b}-\tau+c$ on $[0, \infty)$ and $h(s)=\|\nabla \varphi\|_{\infty, \Omega \times[0, s]}$ on $[0, T]$. Then (3.28) implies

$$
g(h(s)) \geq 0 \text { for each } s \in[0, T] .
$$

Obviously, the function $g$ achieves its minimum value at $s_{0}=\frac{1}{(\varepsilon b)^{\frac{1}{b-1}}}$. The minimum value

$$
\begin{aligned}
g\left(s_{0}\right) & =\frac{\varepsilon}{(\varepsilon b)^{\frac{b}{b-1}}}-\frac{1}{(\varepsilon b)^{\frac{1}{b-1}}}+c \\
& =c-\frac{\varepsilon(b-1)}{(\varepsilon b)^{\frac{b}{b-1}}} \leq-(b-1)
\end{aligned}
$$

provided that

$$
(c+b-1) \varepsilon^{\frac{1}{b-1}} \leq \frac{b-1}{b^{\frac{b}{b-1}}} .
$$

Recall that $\varphi(x, 0)$ satisfies the boundary value problem

$$
\begin{array}{ll}
\Delta \varphi(x, 0)=-\frac{\sigma^{\prime}\left(u_{0}(x, 0)\right)}{\sigma\left(u_{0}(x, 0)\right)} \nabla u_{0}(x, 0) \cdot \nabla \varphi(x, 0) & \text { in } \Omega, \\
\varphi(x, 0)=\varphi_{0}(x, 0) & \text { on } \partial \Omega .
\end{array}
$$

Our assumptions on $u_{0}, \varphi_{0}, \sigma(s)$ imply that the coefficient on the right-hand side of (3.32a) are bounded. We can conclude from (3.26) that $|\nabla \varphi(x, 0)|$ is bounded. Thus we can find the largest $T$ such that (3.7), (3.31), and

$$
h(0)=\|\nabla \varphi(\cdot, 0)\|_{\infty, \Omega} \leq s_{0}
$$

all hold. Denote it by $T_{0}$. Then Lemma 2.3 says that if $h$ is continuous on $\left[0, T_{0}\right]$ we have

$$
\|\nabla \varphi\|_{\infty, \Omega \times\left[0, T_{0}\right]} \leq s_{0} .
$$

Later we will indicate how we can construct a sequence of approximate solutions $\left(u_{\varepsilon}, \varphi_{\varepsilon}\right)$ with $\left|\nabla \varphi_{\varepsilon}\right|$ being bounded. Here we wish to show that if $|\nabla \varphi|$ is bounded then $h$ is continuous, and hence (3.34) holds. To see this, we differentiate (1.1a) with respect to $x_{i}, i=1, \cdots, N$, respectively. We can conclude from the resulting equations that $\nabla u$ is Hölder continuous. Pick a sequence $\left\{t_{n}\right\} \subset[0, T]$ with $\lim _{n \rightarrow \infty} t_{n}=t_{0}$. Define

$$
\begin{aligned}
& \varphi_{n}=\varphi\left(x, t_{n}\right), \\
& \theta(x, t)=-\frac{\sigma^{\prime}(u(x, t))}{\sigma(u(x, t))} \nabla u(x, t) .
\end{aligned}
$$


Then we have

$$
\begin{array}{ll}
\Delta \varphi_{n}=\theta\left(x, t_{n}\right) \cdot \nabla \varphi_{n} & \text { in } \Omega, \\
\varphi_{n}=\varphi_{0}\left(x, t_{n}\right) & \text { on } \partial \Omega .
\end{array}
$$

By a calculation similar to (3.26), we obtain that $\left\{\varphi_{n}\right\}$ is precompact in $C^{1}(\bar{\Omega})$. We can extract a subsequence of $\left\{\varphi_{n}\right\}$, still denoted by $\left\{\varphi_{n}\right\}$, such that

$$
\varphi_{n} \rightarrow \varphi^{*} \quad \text { strongly in } C^{1}(\bar{\Omega}) \text {. }
$$

Pass to the limit in (3.36a)-(3.36b) to get

$$
\begin{array}{ll}
\Delta \varphi^{*}=\theta\left(x, t_{0}\right) \cdot \nabla \varphi^{*} & \text { in } \Omega, \\
\varphi^{*}=\varphi_{0}\left(x, t_{0}\right) & \text { on } \partial \Omega .
\end{array}
$$

By the uniqueness of a solution to the above problem, we have

$$
\varphi^{*}=\varphi\left(x, t_{0}\right)
$$

Consequently, the whole sequence $\left\{\varphi_{n}\right\}$ converges to $\varphi\left(x, t_{0}\right)$ in $C^{1}(\bar{\Omega})$. This completes the proof of (3.34).

We consider $\left(u\left(x, t+T_{0}\right), \varphi\left(x, t+T_{0}\right)\right)$ on $\Omega \times\left[0, T_{0}\right]$. Conditions (3.31) and (3.33) still hold, and so does (3.34). Therefore, we can extend the solution in the time direction as far away as we want.

Existence of a solution can be established via the Leray-Schauder theorem [9, p. 280]. To this end, we define an operator $\mathbb{B}$ from $C\left(\overline{\Omega_{T}}\right)$ into $C\left(\overline{\Omega_{T}}\right)$ as follows: We say $u=\mathbb{B}(v)$ if $v \in C\left(\overline{\Omega_{T}}\right)$ and $u$ is the solution of the problem

$$
\begin{array}{ll}
\partial_{t} u-\Delta u=\sigma(v)|\nabla \varphi|^{2} & \text { in } \Omega_{T}, \\
u=u_{0} & \text { on } \partial_{p} \Omega_{T},
\end{array}
$$

where $\varphi$ solves the boundary value problem

$$
\begin{array}{ll}
-\operatorname{div}(\sigma(v) \nabla \varphi)=0 & \text { in } \Omega_{T} \\
\varphi=\varphi_{0} & \text { on } \Sigma_{T} .
\end{array}
$$

To see that $\mathbb{B}$ is well-defined, we conclude from (1.9) for each $p>1$ there is a positive number $c$ depending on the continuity of $\sigma(v)$ and $\partial \Omega$ such that

$$
\|\nabla \varphi\|_{p, \Omega} \leq c\left\|\nabla \varphi_{0}\right\|_{p, \Omega}
$$

This is more than enough to guarantee that $u$ is Hölder continuous in $\overline{\Omega_{T}}$. Since the two problems in the definition of $\mathbb{B}$ are both linear, we can conclude that $\mathbb{B}$ is continuous and 
maps bounded sets into precompact ones. We still need to show that there is a positive number $c$ such that

$$
\|u\|_{C\left(\overline{\Omega_{T}}\right)} \leq c
$$

for all $u \in \mathbb{B}$ and $\varepsilon \in(0,1)$ satisfying $u=\varepsilon \mathbb{B}(u)$. This equation is equivalent to

$$
\begin{array}{ll}
\partial_{t} u-\Delta u=\varepsilon \sigma(u)|\nabla \varphi|^{2} & \text { in } \Omega_{T}, \\
-\operatorname{div}(\sigma(u) \nabla \varphi)=0 & \text { in } \Omega_{T}, \\
u=\varepsilon u_{0} & \text { on } \partial_{p} \Omega_{T}, \\
\varphi=\varphi_{0} & \text { on } \Sigma_{T} .
\end{array}
$$

To obtain (3.42), we have to apply our early proof to this problem. We only mention that by the calculations in (3.20), (3.41) implies that $|\nabla u| \in L^{\infty}\left(\Omega_{T}\right)$, and thus (3.26) remains valid. Note that (3.41) is only used to justify the regularity of the solution. In particular, the constant $c$ in (3.41) does not appear elsewhere in our proof. We have all the ingredients necessary to conclude (3.42). This finishes the proof of the main theorem.

\section{References}

[1] W. Allegretto, Y. Lin, and S. Ma, Existence and long time behaviour of solutions to obstacle thermistor equations, Discrete \& Continuous Dynamical System-A, 8 (2002), 757-780.

[2] W. Allgretto, Y. Lin, and A. Zhou, A box scheme for coupled systems resulting from microsensor thermistor problems, preprint.

[3] S. N. Antontsev and M. Chipot, The thermistor problem: existence, uniqueness, and blowup, SIAM J. Math. Anal., 25 (1994), 1128-1156.

[4] L. A. Caffarrelli and A. Friedman, Blow-up of solutions of nonlinear heat equations, J. Math. Anal. Appl., 129 (1988), 409-419.

[5] F. Chiarenza, M. Frasca, and P. Longo, $W^{2, p}$-solvability of the Dirichlet problem for nondivergence elliptic equations with VMO coefficients, Trans. Amer. Math. Soc., 336 (1993), 841-853.

[6] G. Cimatti, Remark on existence and uniqueness for the thermistor problem under mixed boundary conditions, Q. Appl. Math., 47 (1989), 117-121.

[7] G. Cimatti and G. Prodi, Existence results for a non-linear elliptic system modelling a temperature dependent electrical resistor, Ann. Mat. Pure Appl., 162 (1992), 33-42.

[8] E. DiBenedetto, Degenerate Parabolic Equations, Springer-Verlag, New York, 1993.

[9] D. Gilbarg and N. S. Trudinger, Elliptic Partial Differential Equations of Second Order, Springer-Verlag, Berlin, 1983.

[10] J. Heinonen, T. Kilpeläinen, and O. Martio, Nonlinear Potential Theory of Degenerate Elliptic Equations, Oxford Mathematical Monographs, Clarendon Press, Oxford, 1993.

[11] S. D. Howison, J. F. Rodrigues, and M. Shillor, Stationary solutions to the thermistor problem, J. Math. Anal. Appl., 174 (1993), 573-588.

[12] V. Hrynkiv and S. Koshkin, Optimal control of a thermistor problem with vanishing conductivity, arXiv:1902.01505.

[13] S. Kutluay, A. R. Bahadir, and A. Özdes, A variety of finite difference methods to the thermistor with a new modified electrical conductivity, Appl. Math. Comput., 106 (1999), 205-213. 
[14] Q. A. Ladyzenskaja, V. A. Solonnikov, and N. N. Ural'ceva, Linear and Quasi-linear Equations of Parabolic Type, Tran. Math. Monographs, Vol. 23, AMS, Providence, RI, 1968.

[15] C. Miao, Weak solution of class of nonlinear heat equation systems and application to the Navier-Stokes system, J. Differential Equations, 61 (1986), 141-151.

[16] J. R. Rodrigues, Obstacle Problems in Mathematical Physics, North-Holland Math. Studies, Vol. 134, North-Holland, Amsterdam, 1987.

[17] A. N. Sandjo, S. Moutari, and Y. Gningue, Solutions of fourth-order parabolic equation modeling thin film growth, J. Differential Equations, 259 (2015), 7260-7283.

[18] E. M. Stein, Harmonic Analysis: Real-Variable Methods, Orthogonality, and Oscillatory Integrals, Princeton University Press, Princeton, NJ, 1993.

[19] A. S. Wood and S. Kutluay, A heat balance integral model of the thermistor, Int. J. Heat Mass Transfer, 38 (1995), 1831-1840.

[20] X. Xu, Local partial regularity theorems for suitable weak solutions of a class of degenerate systems, Appl. Math. Optim., 34 (1996), 299-324.

[21] X. Xu, Partial regularity of solutions to a class of degenerate systems, Trans. Amer. Math. Soc., 349 (1997), 1973-1992.

[22] X. Xu, A local partial regularity theorem for weak solutions of degenerate elliptic equations and its application to the thermistor problem, Differential Integral Equations, 12 (1999), 83100.

[23] X. Xu, Local and global existence of continuous temperature in the electrical heating of conductors, Houston J. Math., 22 (1996), 435-455.

[24] X. Xu, Logarithmic upper bounds for weak solutions to a class of parabolic equations, Proc. Roy. Soc. Edinburgh Sect. A, 149 (2019), 1481-1491.

[25] X. Xu, On the existence of bounded temperature in the thermistor problem with degeneracy, Nonlinear Anal., TMA, 42 (2000), 199-213.

[26] X. Xu, Global existence of strong solutions to a biological network formulation model in $2+1$ dimensions, Discrete Contin. Dyn. Syst. Ser. A, 40 (2020), 6289-6307.

[27] X. Xu, Local and global existence of solutions to a fourth-order parabolic equation modeling kinetic roughening and coarsening in thin films, Commun. Math. Sci., 15 (2017), 2195-2218.

[28] X. Xu, Exponential integrability of temperature in the thermistor problem, Differential and Integral Equations, 17(2004), 571-582.

[29] X. Xu, Nonlinear diffusion in the Keller-Segel model of parabolic-parabolic type, J. Differential Equations, 276 (2021), 264-286.

[30] G. Yuan, Regularity of solutions of the thermistor problem, Appl. Anal., 53 (1994), 149-156. 